\title{
Genome-wide association mapping for root cone angle in rice
}

\author{
Mathilde Bettembourg ${ }^{1}$ D, Audrey Dardou', Alain Audebert ${ }^{1,2}$, Emilie Thomas', Julien Frouin?', \\ Emmanuel Guiderdoni ${ }^{1}$, Nourollah Ahmadi ${ }^{1}$, Christophe Perin ${ }^{1}$, Anne Dievart ${ }^{1,3}$ and Brigitte Courtois ${ }^{1 *}$
}

\begin{abstract}
Background: Plant root systems play a major role in anchoring and in water and nutrient uptake from the soil. The root cone angle is an important parameter of the root system architecture because, combined with root depth, it helps to determine the volume of soil explored by the plant. Two genes, DRO1 and SOR1, and several QTLs for root cone angle have been discovered in the last 5 years.

Results: To find other QTLs linked to root cone angle, a genome-wide association mapping study was conducted on two panels of 162 indica and 169 japonica rice accessions genotyped with two sets of SNP markers (genotypingby-sequencing set with approximately 16,000 markers and high-density-rice-array set with approximately 300,000 markers). The root cone angle of all accessions was measured using a screen protractor on images taken after 1 month of plant growth in the Rhizoscope phenotyping system. The distribution of the root cone angle in the indica panel was Gaussian, but several accessions of the japonica panel (all the bulus from Indonesia and three temperate japonicas from Nepal or India) appeared as outliers with a very wide root cone angle. The data were submitted to association mapping using a mixed model with control of structure and kinship. A total of 15 QTLs for the indica panel and 40 QTLs for the japonica panel were detected. Genes underlying these QTLs (+/-50 kb from the significant markers) were analyzed. We focused our analysis on auxin-related genes, kinases, and genes involved in root developmental processes and identified 8 particularly interesting genes.
\end{abstract}

Conclusions: The present study identifies new sources of wide root cone angle in rice, proposes ways to bypass some drawbacks of association mapping to further understand the genetics of the trait and identifies candidate genes deserving further investigation.

Keywords: Rice, Oryza sativa, Indica, Japonica, root cone angle, hydroponics, association mapping, GWAS

\section{Background}

Roots are organs that play several essential roles in plants. Roots are needed to uptake soil water and mineral nutrients and anchor plants to the ground. In rice, several ideotypes of root architecture have been proposed depending on the relative importance given to these different roles. A root system with deep and thick roots and a high root to shoot ratio is needed to enable a better uptake of water, which is found at depth under water stress conditions, (Gowda et al. 2011; Fukai and Cooper 1995). Conversely, for nutrient uptake, ideotypes differing in root depth, number and angle were recommended for immobile

\footnotetext{
* Correspondence: brigitte.courtois@cirad.fr

${ }^{1}$ Cirad, UMR AGAP, F34398 Montpellier Cedex 5, France

Full list of author information is available at the end of the article
}

nutrients such as phosphorus, which are dominantly located in the cultivated layer of soil, and for nutrients easily soluble in the soil solution and more prone to leaching and diffusion in the profile such as nitrogen (Lynch and Brown, 2001; White et al. 2013). Obviously, a combination of both shallow and deep roots, if physiologically and genetically possible in terms of resource allocation, would be the best option to enable optimal topsoil and subsoil exploration.

Root ideotypes should integrate functional parameters such as water and nutrient use efficiency and plasticity in addition to root architecture, as suggested by Ahmadi et al. (2014). In rice, which can be grown under fully anaerobic conditions (irrigated ecosystems), fully aerobic conditions (upland ecosystems) and conditions alternating between aerobic and anaerobic (rainfed lowland 
ecosystems), roots play an additional role in oxygen transport to the root tips. Good oxygenation is generally obtained through the development of aerenchyma in the cortex via an apoptosis mechanism but it has been suggested that, in some cases, aerial roots can develop above the soil to provide oxygen to the roots under the soil (Ueno and Sato 1989).

The spatial distribution of roots in a soil profile is determined by a combination of root growth angle and root length (Abe and Morita 1994). In this paper, the root cone angle designates the angle relative to a vertical axis (Fig. 1). Root cone angle and root depth do not always correlate. In some studies, plants with a narrow root cone angle tended to have increased root depth (Oyanagi et al. (1993) in bread wheat and Kato et al. (2006) in rice) while in other studies, this correlation was absent (Sanguineti et al. (2007) in durum wheat), weak (Uga et al. (2009) in rice), or depended on the soil hydrology and plant growing conditions (Abe and Morita (1994) and Kundur et al. (2015) in rice). While the depth of a root system is particularly complicated to determine, the root cone angle is reasonably easy to measure using specific phenotyping systems such as the basket method (Oyanagi et al. 1993) or the shovelomics method in the field (Trachsel et al. 2011). The broadsense heritability of root cone angle is moderate to high in cereals so it should be possible to get good responses to selection for this trait (Sanguinetti et al. 2007; Singh et al. 2012). Because the root cone angle could be an important trait to increase crop production under stress conditions (Uga et al. 2015), its natural phenotypic variability and genetic control need to be known.

Large genetic variability has been observed in rice (Oryza sativa L.) for different root traits such as root

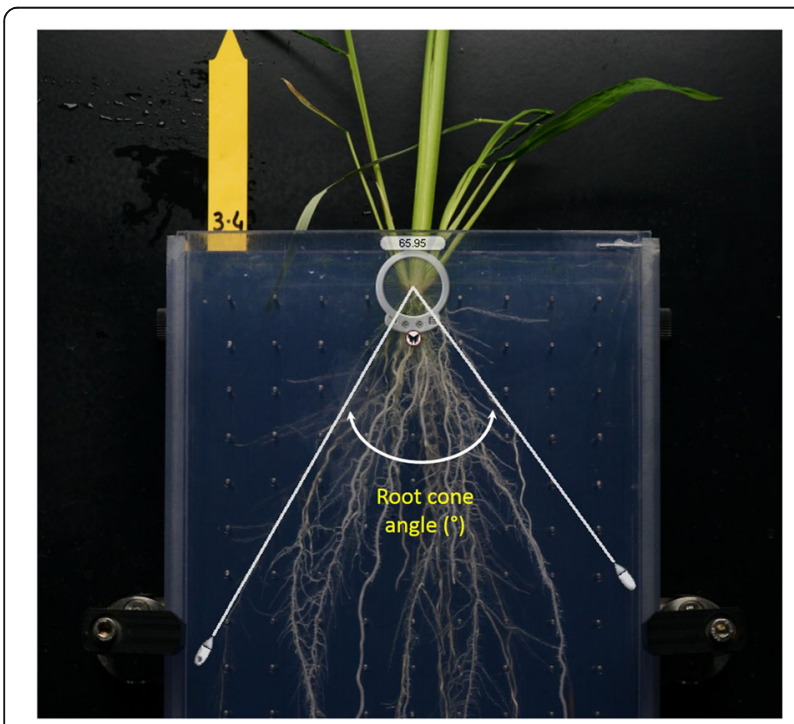

Fig. 1 Measurement of the root cone angle with a screen protractor depth, root thickness and root-to-shoot ratio (O'Toole and Bland, 1987). This variability partly follows the genetic organization of the species, which is subdivided into two major components: the indica and japonica subspecies, which themselves are subdivided into varietal groups and ecotypes within varietal groups (Garris et al. 2005). An ecotype is often characterized by a specific combination of root characteristics that are generally related to its hydrological adaptation (Lafitte et al. 2001). Of course, the ecotype general trends can largely be modified by soil conditions (composition, $\mathrm{pH}$, impedance), soil biology (presence of mycorrhizae or nematodes), water regimes (aerobic or anaerobic or alternating between the two conditions) and genotype $\mathrm{x}$ environment interactions. The root cone angle has seldom been considered among the studied architectural root traits, except by Japanese teams who showed that most accessions of some ecotypes (aman, boro and some of the aus varieties within the indica subspecies; bulu within the japonica subspecies) had a high proportion of crown roots growing horizontally (Ueno and Sato 1992; Kato et al. 2006; Uga et al. 2009). They correlated a wide root cone angle with an absence of response to a gravitropic stimulus (Ueno and Sato 1992).

Several teams have studied the genetic control of root cone angle and related traits in rice (Norton and Price 2009; Uga et al. 2012, 2013, 2013, 2015, 2015; Hanzawa et al. 2013; Kitomi et al. 2015; Lou et al. 2015). The detected QTLs and genes are positioned on Fig. 2 and summarized in Additional file 1: Table S1. At least six QTLs named DRO1 (for DEEP ROOTING 1) to DRO5 were shown to determine the ratio of deep rooting, which is an index based on the frequency of high root growth angles, in a population of approximately 100 recombinant inbred lines (RILs) derived from an indica $x$ japonica cross between IR64 and Kinandang Patong (Uga et al. 2011). Phenotyping was performed using the basket method. Kinandang Patong was the deep-rooted parent with a narrow cone angle. DRO1, the major QTL located on chromosome (chr) 9, was cloned (Uga et al. 2011; Uga et al. 2013). The DRO1 gene (Os09g0439800) was shown to be involved in the root response to gravitropism, downstream of the auxin signaling pathway. The four other QTLs have been fine mapped but not yet cloned (Uga et al. 2013, Uga et al. 2015, Kitomi et al. 2015). Another cross between Gemdjah Beton, a tropical japonica Indonesian variety from the bulu ecotype, and Sasanishiki, a temperate japonica accession from Japan, was used to detect a major QTL named qSOR1 involvedk in soil surface rooting on chr 7, with other minor QTLs on chr 3, 4 and 6 (Uga et al. 2012). One gene called SOR1 (for SOIL-SURFACE ROOTING 1) was isolated from a mutant population of Nipponbare (Hanzawa et al. 2013). This gene (Os04g0101800) was located on chr 4. A deletion in this gene, which is probably 


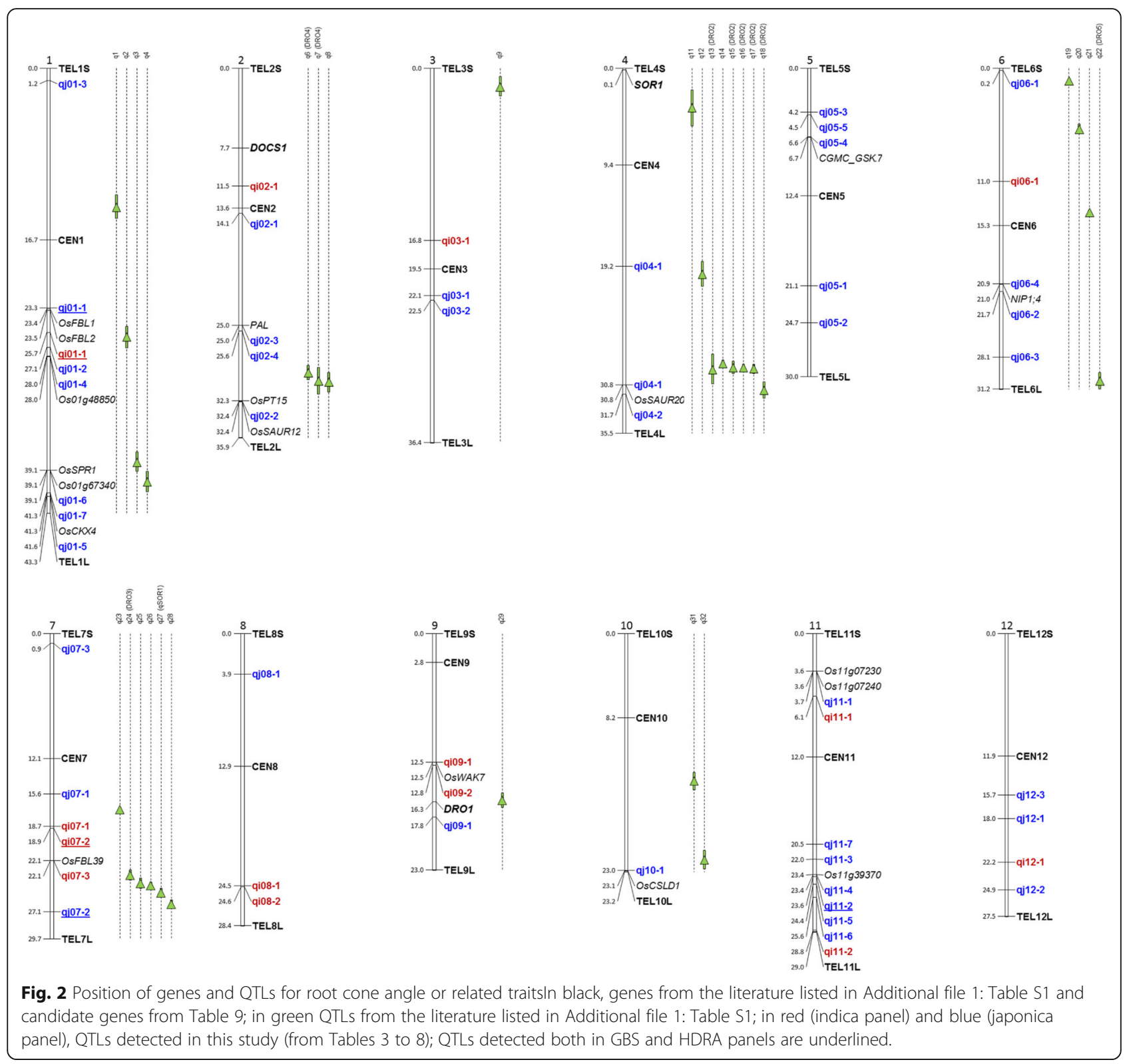

involved in auxin signaling, induced a loss of the gravitropic response in young plants and a soil-surface rooting phenotype.

Several genes or QTLs for root cone angle have been discovered in the last five years, but many remain to be found since the trait appears to be controlled by a combination of genes carrying alleles with major effects, such as the DRO1 allele from Kinandang Patong, and alleles with smaller effects. Genome-wide association study (GWAS) in natural populations is an efficient tool in rice for localizing QTLs faster and with a much higher resolution than classical genetic approaches using mapping populations (Zhang et al. 2016). GWAS requires a high marker density that is now easily accessible due to GBS (genotyping by sequencing) as described by
Elshire et al. (2011) or to the re-sequencing of the rice genome and the discovery of millions of singlenucleotide polymorphisms (SNPs) (Alexandrov et al. 2015). GWAS allows for a precise localization of QTLs due to the rapid decay of linkage disequilibrium (LD) in the association panels. However, rice incurs a high risk of false-positive tests during GWAS because of the bipolar genetic structure of the species. This structural effect can be corrected by using panels specific to each subspecies and by using models that consider subpopulation structure and kinship. Several large panels of rice varieties genotyped with high marker density have been developed for GWAS (e.g., Huang et al. (2010), Zhao et al. (2011), McCouch et al. (2016)). A panel called Orytage composed of 170 japonica rice accessions and 204 indica 
rice accessions has been developed by Cirad (Courtois et al. 2013, Lafarge et al. 2017). This panel has been genotyped by GBS and approximately 16,000 markers were detected (SNP and diversity arrays technology (DArT) markers) in the japonica panel (Courtois et al. 2013) and a similar number in the indica panel (Lafarge et al. 2017). The High-Density Rice Array (HDRA) developed by McCouch et al. (2016) is composed of more than 1500 inbred rice varieties of Oryza sativa genotyped with 700,000 SNPs. A very large proportion of the accessions belonging to the Orytage panel are also included in the HDRA panel. The Orytage japonica panel has been used for GWASs on several root traits, including root cone angle (Courtois et al. 2013). However, in this early study, the range of variability in root cone angle was narrowed by trigonometrically deducing this angle from segment measurements and not directly measuring it. Other panels have been used for GWASs for root architectural traits in which root depth was analyzed but not the root cone angle itself (Lou et al. 2015; Phung et al. 2016).

In this study, we used the phenotypic data obtained by directly measuring the root cone angle for sub-samples of the indica and japonica Orytage panels. We conducted GWAS using primarily the GBS data but also the HDRA SNP data. To identify candidate genes associated with the significant markers, we conducted a survey of the genes present in the vicinity of the significant markers with a particular interest given to auxin- and kinases-related genes because genes of these families have already been shown to play a role in determining root cone angle.

\section{Methods}

\section{Plant materials}

A sub-sample of the Orytage panel was used in this study. The indica panel was composed of 162 accessions. The japonica panel was composed of 169 accessions. The seeds were obtained either from the Centre de Ressources Biologiques Tropicales de Montpellier (http://golo.cirad.fr/FR/) or from the International Rice Research Institute (IRRI) gene bank. The accessions were seed-increased by single-seed-descent over two generations in a Cirad greenhouse. The list of accessions with their country of origin and seed source is given in Additional file 2: Table S2 for the indica panel and Additional file 3: Table S3 for the japonica panel.

\section{Genotyping by GBS and filtration of the resulting data}

Both panels were genotyped by GBS. The GBS data were available for 156 indica and 166 japonica accessions. Detailed information on the genotyping procedure is given in Lafarge et al. (2017) for the indica panel and Courtois et al. (2013) for the japonica panel. Genotyping was conducted at Diversity Arrays Technology Pty Ltd. (DArT P/
L), Australia. Briefly, this method involved a step of genome complexity reduction using PstI/TaqI restriction enzymes followed by Illumina short-read sequencing of the restricted products. The resulting sequences, which were trimmed to $69 \mathrm{bp}$, were aligned to the Nipponbare sequence to determine the position of the restriction sites (DArT markers) and the position of the polymorphisms within the 69-bp sequence (SNP markers). Sequences with no or several positions on the genomes were excluded. Markers with a rate of missing data above 20\%, a heterozygosity above $10 \%$ or a minor allele frequency (MAF) below $2.5 \%$ were discarded. The few remaining heterozygotes (approximately 1.5\%) were set as missing data. The missing data were then imputed using Beagle v3.3 (Browning and Browning 2007). The final indica and japonica sets had 16,232 and 15,921 markers, respectively.

Structure was analyzed on each panel using the program Structure (Pritchard et al. 2000), and the results suggested the existence of 4 subpopulations (I1 to I4) in the indica panel (Lafarge et al., 2017) and 6 subpopulations (J1 to J6) in the japonica one (Courtois et al. 2013). An accession was assigned to a sub-population when more than $80 \%$ of its genome came from that subpopulation; otherwise it was classified as admixed (m). For each panel, an unweighted neighbor-joining (NJ) tree based on a dissimilarity matrix computed using a shared allele index was built using DARwin software (Perrier and Jaquemout-Collet 2006) and the subpopulation attributions of the accessions were projected as colors on the tree (Additional file 4: Figure S1 and Additional file 5: Figure S2). For the indica panel (Additional file 4: Figure S1), I1 (69 acc.) included traditional indica varieties from Asia; I2 (45 acc.) included improved irrigated or upland indica varieties; I3 (16 acc.) corresponded to a special group of indica accessions from Madagascar grown at moderate to high elevation (1250 to $1750 \mathrm{~m}$ ), and I4 included a small set (5 acc.) of aus or boro accessions from eastern India. The last 21 accessions were classified as admixed. For the japonica panel (Additional file 5: Figure S2), J1 included a large set (46 acc.) of improved varieties from Africa, Latin America and Madagascar; J2 (19 acc.) included improved varieties from South-East Asia; J3 included a small set (5 acc.) of improved varieties derived from Colombia 1; J4 (8 acc.) was composed of lowland bulus from Indonesia; J5 (30 acc.) consisted of upland varieties from equatorial Asia, and J6 (10 acc.) comprised temperate accessions from high elevation or high latitudes. The last 48 accessions were classified as admixed. The sub-population attributions are given in Additional file 2: Table S2 and Additional file 3: Table S3.

\section{Genotyping by HDRA}

The HDRA data were recovered from https://ricediversity.org/data/. A subset of accessions corresponding to 
those used in this study was extracted. The corresponding HDRA ID is given in Additional file 2: Table S2 and Additional file 3: Table S3. For several accessions (35 for the indica panel and 20 for the japonica panel), HDRA data were not available. The smaller HDRA panel size explains why the GBS data were used as priority and why the two datasets were not merged. The extracted dataset was submitted to the same filtering as were the GBS data, notably to remove markers that became monomorphic within each panel. The missing data were imputed using Beagle v4. The final dataset was composed of 337,150 and 302,942 SNP markers for the indica and japonica panels, respectively. Few markers were shared with the GBS dataset. All four datasets under HapMap format (indica and japonica GBS and indica and japonica HDRA) are available for download at http://tropgenedb.cirad.fr/tropgene/JSP/interface.jsp?module=RICE under study type "genotypes" as a zip file named "Root cone angle".

To address discrepancies in names and genotypes between the two genotypic datasets due to differences in seed samples used for the genotyping, a subset of 2000 markers was randomly chosen in each dataset, and two pairs of NJ trees were constructed independently. The GBS and HDRA diversity trees obtained were compared for both subspecies. This procedure enabled the identification of a few accessions that were not classified in the same subspecies or were clearly not the same in the GBS and HDRA panels either because the initial sample was not identical or was heterogeneous or because the sample drifted during the multiplication phase at one site or the other. Since phenotyping was performed with the same seed samples that were used for GBS, these accessions were discarded from the HDRA panels, reducing the size of the HDRA panels to 128 indica accessions and 143 japonica accessions with both phenotypic and genotypic data. The reduction in panel size did not modify the panel structure, and all subpopulations detected in the GBS panels were present in the HDRA panels.

\section{Phenotyping}

The two panels were phenotyped in the Rhizoscope phenotyping system, which is described in detail by Courtois et al. (2013). Briefly, the plants were grown in $50 \times 20 \times 2 \mathrm{~cm}$ homemade Plexiglas rhizoboxes (internal dimensions) filled with glass beads $5 \mathrm{~mm}$ in diameter. Each plate contained a grid of nails that helped maintain the root system in place during all operations. The rhizoboxes were set in tanks (48 rhizoboxes per tank) in which an aerated nutrient solution (modified Hoagland solution; $\mathrm{pH}$ 5.4) was circulated. The conditions in the growth chamber were $28{ }^{\circ} \mathrm{C}$ during the day and $25{ }^{\circ} \mathrm{C}$ at night with a 12:12 photoperiod. The radiation was 400 to $450 \mu \mathrm{mol}$ photons per $\mathrm{m}^{2}$ per $\mathrm{s}$ (PAR). The relative humidity was set to $55 \%$. One healthy pre-germinated seed was set on each rhizobox on the top of the beads and allowed to grow. After 28 days of growth, the experiment was stopped. The glass beads were removed from the rhizoboxes, and a high-definition image of the plate with the plant in position was taken. In comparison with the method used by Courtois et al. (2013), the imaging system was set in an aluminum frame enabling all images to be taken from the same distance and with the same magnification. The angle of the root cone to the vertical axis was measured in degrees using a screen protractor (Screen protractor v4.0, iconico.com). This device enabled the rapid measurement of the root cone angle on an image by positioning the center of the seethrough visor on the basis of the tillering plateau and aligning the two mobile branches of the protractor to the two most external left and right crown roots (Fig. 1). A few plants that had accidentally been moved from their rhizoboxes during harvest were not measured.

For both panels, the experimental designs were alpha lattices with two replications, and the tank was considered a block effect within replication. The experimental unit was one rhizobox. Since the phenotyping system could only handle 192 plants at once and since $3 \times 2$ replicated controls (the indica variety IR64 and the japonica variety Azucena) were included in each tank, the replications were grown at two-month intervals.

\section{Statistics}

ANOVA was conducted on the experimental data using a mixed model considering the tank effect to be random. Lsmeans were computed for each accession using SAS v9.2 (SAS Institute, Cary NC, USA). In addition, for each panel, ANOVA was conducted on the accession lsmeans, using sub-populations as the source of variation to assess to what extent the root cone angle was linked to population structure. The admixed accessions were excluded from this specific analysis.

\section{Association analyses}

For all panels, GWAS was conducted with control of kinship among accessions and panel sub-structure using Tassel v5 (Bradbury et al. 2007). As by Courtois et al. (2013), a comparison was first conducted between models with kinship alone and kinship and structure together using an Akaike Information Criterion. The conclusion was that the kinship and structure model best fit the data (data not shown).

For analyses involving the GBS datasets, the percentages of admixture derived from previous analyses (as explained above) were used to control population structure. The kinship matrix was computed using the centered identity-by-state method. Qq (quantile by 
quantile) plots and Manhattan plots were obtained from Tassel. A threshold of $1 \mathrm{e}-04$ was chosen to declare a marker significant for the GBS datasets. As a comparison, Bonferroni correction would lead to a threshold of approximately $5 \mathrm{e}-06$, and the number of independent tests (Gao 2011) computed using the simpleM program would lead to a threshold of $1 \mathrm{e}-05$ for both panels. To confirm that the chosen threshold was reasonable, the q-values corresponding to the false discovery rates (FDRs) were also computed for each of the significant markers using the formula $\mathrm{N}^{*} \mathrm{Pmax} / \mathrm{n}$, where $\mathrm{N}$ was the total number of markers in the dataset, Pmax was the observed $p$-value and $\mathrm{n}$ was the number of markers significant at this threshold (Storey and Tibshirani 2003). Markers that were significant in an interval of less than $+/-50 \mathrm{~kb}$ were considered to belong to the same QTL.

For analyses involving the HDRA datasets, the number of accessions was $20 \%$ and $13 \%$ lower than for the GBS data for the indica and japonica panels, respectively. This difference was important and justified repeating the structure analysis. Principal Component Analyses (PCA) were therefore conducted on the genotypic data; 4 and 6 axes were retained for the indica and japonica panels, respectively, since the number of sub-populations was unchanged compared to that of the GBS data. A threshold of 5e-06, which considers the fact that the HDRA datasets included approximately 20 times more markers than did the GBS datasets, was chosen to declare a QTL significant. By comparison, Bonferroni correction and the number of independent tests would lead to thresholds of approximately $1 \mathrm{e}-07$ and $5 \mathrm{e}-07$, respectively. The q-values were computed the same way as for the GBS datasets.

In GWAS, a low sample size relative to the number of markers and association tests can lead to inconsistency in the results. To assess the robustness of the detected associations, a sub-sampling procedure was conducted for the GBS data. The method is similar to that used by Tian et al. (2011) on maize except that our association analysis, like that of Lafarge et al. (2017), was based on a single marker model rather than a multiple marker model. A set of $80 \%$ of the accessions (125 accessions for the indica panel and 133 for the japonica panel) was chosen at random without replacement and GWAS was conducted on this set. Random sub-sampling was repeated 100 times. The number of times that an association was detected at $p<1 \mathrm{e}-03$ was counted to obtain a sub-sampling posterior probability for each marker. Based on the distribution of the results, a threshold that had a 95\% chance of not being overtaken was chosen. Sub-sampling was preferred to bootstrap because of the genetic structure of the panels and the simplicity in file preparation from not having several copies of a given accession. For the HDRA dataset, because of the smaller size of the panel and the much higher number of markers considerably slowing down the analyses, no robustness test was attempted.

\section{Detection of candidate genes}

The position of the significant markers was compared to that of approximately 200 root-related genes in rice from a database established in the framework of the EURoot project (http://euroot.cirad.fr/euroot/JSP/interface.jsp?module $=$ RICE). Then, the function of the candidate genes in which significant markers were detected was determined using OrygenesDB (http://orygenesdb.cirad.fr/). In addition, genes located in intervals of $50 \mathrm{~kb}$ on both sides of the marker were explored. This window size was chosen because it was intermediate between the average marker distance (approximately $25 \mathrm{~kb}$ in the GBS datasets and $1.5 \mathrm{~kb}$ in the HDRA datasets) and the distance at which LD decayed to half its initial level (estimated at $100 \mathrm{~kb}$ for the indica panel and $150 \mathrm{~kb}$ for the japonica panel on average across all chromosomes but much more variable in short-range segments). Particular attention was paid to auxin-related genes and to kinases because the cloned genes for root angle belonged to these families. Among kinases, leucine-rich repeat receptor-like kinases $(L R R-R L K s)$ were identified using a list of $L R R-R L K$ genes in rice and a nested list of $L R R-R L K S$ involved in root development established by Dievart et al. (2016) and Fischer et al. (2016).

\section{Results}

Analyses of variance conducted on the root cone angle in different experiments showed that the genotype effect was highly significant for both panels. The mean root cone angle of the two repeated controls (IR64 and Azucena) was similar in the two experiments, with slightly higher values in the experiments involving the japonica panel; this similarity enabled a comparison of the distribution of frequency between the two panels (Fig. 3). The indica accessions recorded lower values (range from 21.8 to $93.0^{\circ}$ ) than did the japonica accessions (range 36.3 to $164.4^{\circ}$ ), whose distribution was shifted towards higher values (Table 1). There was discontinuity in the distribution of the japonica accessions, with 10 accessions with a very wide root cone angle (above $115^{\circ}$ ). Examples of extreme accessions are shown in Fig. 4. Seven of these accessions (Bulu Pandak, Cicih Beton, Gundil Kuning, Molok, Padi Boenar, Poenoet Hitam, and Reket Maun) are from Indonesia and encompass all the accessions of subpopulation J4, which corresponds to the bulu ecotype (a special group of rainfed lowland accessions). Three accessions (Gompa2, Jumula 2 and Kakani 2) are from India and Nepal and belong to subpopulation J6, which is composed of temperate japonica accessions. Conversely, the seven other accessions from subpopulation J6 


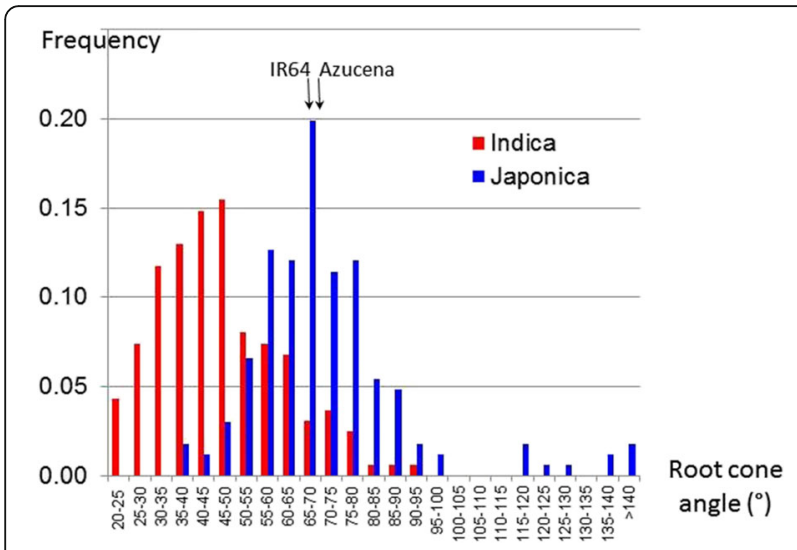

Fig. 3 Distribution of the root cone angle $\left(^{\circ}\right)$ for the indica (red) and japonica (blue) panels

had very acute root cone angles (from 46.9 to $69.9^{\circ}$ ). When the accessions with very wide root cone angles were removed, the mean of the japonica panel dropped to $67.3^{\circ}$ (range 36.3 to $96.0^{\circ}$ ), which was still higher than that of the indica panel.

When undertaking GWAS, a situation in which the phenotypic organization closely parallels the genetic structure is risky since true functional associations can be excluded because these associations are confounded with subpopulation effect. The means of the different subpopulations were computed to assess this risk (Table 2). For the indica panel, subpopulation I4 composed of aus/boro accessions was different from the other three subpopulations, showing a narrower root cone angle. For the japonica panel, subpopulations J4 (bulus) and J3 (a group of improved upland rice varieties derived from Colombia 1) were significantly different from the other subpopulations, and exhibited contrasting behaviors: J4 with the largest root cone angle and J3 with the narrowest one. Subpopulation J6, in which extremes were pooled, was not significantly different from the three other intermediate subpopulations, probably because of its large variance.

Table 1 Statistics of the indica and japonica panels for the root cone angle

\begin{tabular}{lll}
\hline Statistics & Indica panel & Japonica panel \\
\hline No. observations & 162 & 166 \\
Minimum & 21.8 & 36.3 \\
Maximum & 93.0 & 164.4 \\
Mean & 46.4 & 71.5 \\
Standard deviation & 14.5 & 19.7 \\
CV of the panel & 0.31 & 0.27 \\
Mean IR64 & 65.9 & 68.9 \\
Mean Azucena & 67.8 & 70.1 \\
\hline
\end{tabular}

\section{GWAS on the indica panel}

An initial GWAS analysis was conducted on the indica panel using a mixed model with control of structure and kinship (Table 3). This model enabled the control of false positives as seen on the q-q plot (Additional file 6: Figure S3). This analysis enabled the identification of 8 significant markers $(p<1 \mathrm{e}-04)$, corresponding to 5 different QTLs on chr 3, 6, 8 and 9 (Additional file 7: Figure S4). The q-value of these markers varied between 0.01 and 0.16 (Table 3), which can be deemed reasonable and justifies the chosen threshold. Then, the GWAS was repeated using the same model on 100 subsamples, enabling the computation of a posterior probability (the number of times the marker was considered significant in the 100 subsamples). The distribution of the posterior probabilities showed that no marker had greater than a $5 \%$ chance to be detected more than 30 times; therefore, a posterior probability of 0.30 was chosen as a threshold. Based on this criterion, 6 of the 8 original significant markers passed the threshold (posterior probabilities of 0.54 to 0.87 ). The two markers that did not pass the threshold were also those with the lowest initial probabilities. They were detected only 10 to 17 times. The subsampling also enabled the identification of 6 additional markers corresponding to 5 QTLs that were not detected by the full-size panel initial association analysis. Their original $p$-value, which is indicated in italics in Table 3, varied from $1.69 \mathrm{e}-03$ to $1.02 \mathrm{e}-04$. With the exception of q06-1, the MAF of all 14 of these markers was greater than $5 \%$.

Then, a GWAS was conducted on the HDRA panel (more markers but fewer accessions). The analysis enabled the identification of 9 markers $(p<5 \mathrm{e}-06)$ corresponding to 7 QTLs (Table 4). Two of these QTLs (q01-1 on chr 1 and q07-2 on chr 2) corresponded to QTLs identified using the GBS data. These two QTLs were also those with the highest MAF. The other significant markers displayed a low MAF (4 to 7\%). For these markers with low MAF, because of the very large size differences between the two genotypic classes, the tests based on ANOVA had a greater risk of being biased due to variance heterogeneities and of associations being spurious.

\section{GWAS on the japonica panel}

Because of the discontinuity in the distribution of root cone angle within the japonica panel, data were first analyzed as the indica panel and then subjected to additional analyses. In the first analysis conducted on the whole japonica panel (Table 5), the model, although involving both structure and kinship corrections, did not allow for a good control of false positives, as seen in the q-q plot (Additional file 8: Figure S5). Since the distribution was skewed with a long tail in the positive direction, we attempted a log transformation of the data. The 


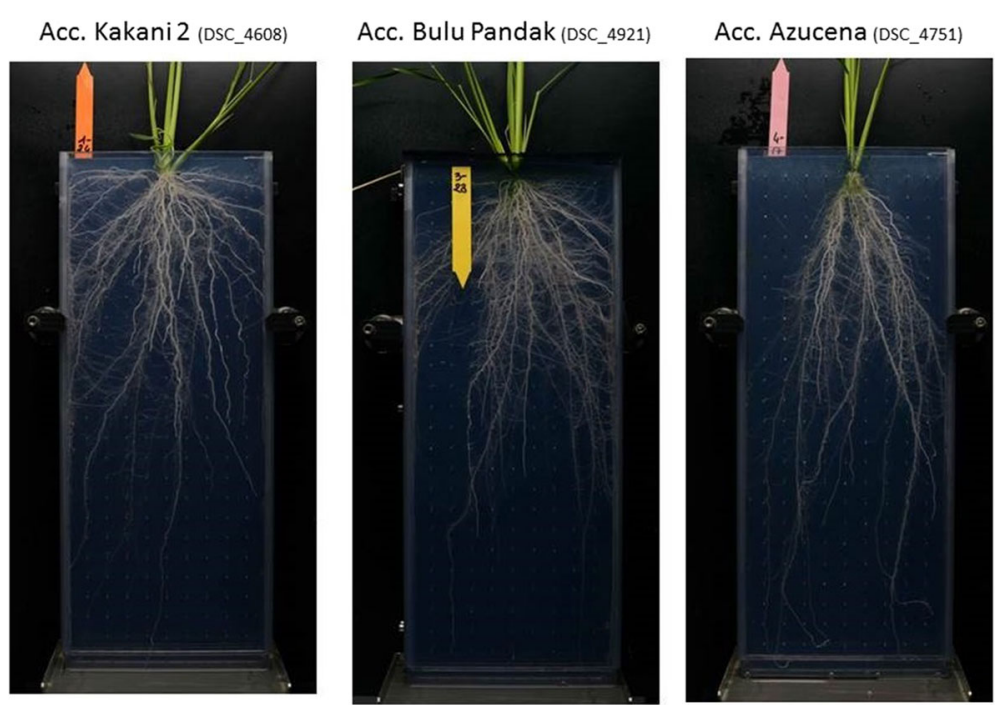

Fig. 4 Examples of root cone angle variation within the japonica panel. Kakani 2 and Bulu Pandak belong to the bulu ecotype and Azucena (check) is a tropical japonica

transformation improved the normalization of the model residuals and decreased the significance of the associations but identified the same associations.

Among the significant markers, several markers had exactly the same $\mathrm{F}$ and $P$ values and, upon verification, were exhibiting exactly the same genotypic pattern, which corresponded to a situation of complete LD $\left(r^{2}=1\right)$. These markers were pooled as intervals in Table 5. However, several markers on different chromosomes were also in complete LD: this was the case for 8

Table 2 Mean per subpopulation (admixed excluded)

\begin{tabular}{|c|c|c|c|c|}
\hline Sub-population & N & \multicolumn{2}{|c|}{$\begin{array}{l}\text { Root cone } \\
\text { angle }\end{array}$} & Subpopulation description \\
\hline \multicolumn{5}{|l|}{ Indica } \\
\hline 11 & 69 & 42.6 & a & Traditional irrigated varieties \\
\hline 12 & 45 & 52.9 & a & $\begin{array}{l}\text { Improved varieties (irrigated } \\
\text { and upland) }\end{array}$ \\
\hline 13 & 16 & 50.2 & a & $\begin{array}{l}\text { Specific group of medium } \\
\text { elevation from Madagascar }\end{array}$ \\
\hline 14 & 5 & 30.0 & $b$ & $\begin{array}{l}\text { Aus/boro varieties from eastern } \\
\text { India }\end{array}$ \\
\hline \multicolumn{5}{|l|}{ Japonica } \\
\hline$J 4$ & 8 & 126.1 & a & $\begin{array}{l}\text { Rainfed lowland bulus from } \\
\text { Indonesia }\end{array}$ \\
\hline 16 & 10 & 82.2 & $b$ & Irrigated temperate rice varieties \\
\hline$J 2$ & 19 & 77.6 & $b$ & Upland rice varieties from SE Asia \\
\hline$J 5$ & 30 & 69.9 & $b$ & $\begin{array}{l}\text { Upland rice varieties from } \\
\text { equatorial Asia }\end{array}$ \\
\hline J1 & 46 & 67.6 & $b$ & $\begin{array}{l}\text { Upland rice acc. From Africa, } \\
\text { Latin America and Madagascar }\end{array}$ \\
\hline$J 3$ & 5 & 53.2 & c & $\begin{array}{l}\text { Improved upland rice varieties } \\
\text { derived from Colombia } 1\end{array}$ \\
\hline
\end{tabular}

markers in an interval between 32.3 and $35.1 \mathrm{Mb}$ on chr 2, for 9 markers in an interval between 3.2 and $6.9 \mathrm{Mb}$ on chr 6 and for 5 markers in an interval between 11.0 and $11.7 \mathrm{Mb}$ on chr 10. This phenomenon can also be seen on the q-q plots and Manhattan plots (straight line at a given probability). These markers with a low MAF discriminated 5 accessions from the rest of the population. These accessions were Gompa 2, Jumula 2 and Kakani 2 (the 3 accessions from Nepal with a wide root cone angle from temperate subpopulation J6), as well as Jumali from Nepal and Lambayque1 from Peru (two admixed accessions with a root cone angle close to the mean of the panel). These last two accessions showed percentages of admixture that did not enable their classification into subpopulation J6; however, for Jumali, this percentage (0.76 in subpopulation J6) was very close to the 0.80 threshold, and for Lambayque1, these percentages (0.60 in subpopulation J6 and 0.33 in subpopulation J4) showed that the accession was intermediate between subpopulation J6 and bulu subpopulation J4. Such markers carried by different chromosomes but in complete LD could not be used for GWAS. Once removed, 13 significant markers corresponding to 12 QTLs remained (Table 5; Additional file 9: Figure S6). Most markers had a low MAF.

Then, as was performed for the indica panel, GWAS was repeated using the same model on 100 subsamples enabling the computation of a posterior probability. In this case as well, the markers that were in complete LD but located on different chromosomes were removed. Based on the distribution of the posterior probabilities, a threshold probability of 0.50 was chosen. Eight of the 12 original significant markers passed the threshold 
Table 3 Markers significant in the GBS indica panel

\begin{tabular}{|c|c|c|c|c|c|c|c|}
\hline Marker & QTL & Chr & Position & Posterior probability & $p$-value & $q$-value & $\begin{array}{l}\text { Number obs } \\
\text { minor allele }\end{array}$ \\
\hline S01_25684060 & qi01-1 & 1 & 25684060 & 0.42 & $1.69 E-03$ & & 46 \\
\hline S01_25685951 & & 1 & 25685951 & 0.41 & $1.73 E-03$ & & 45 \\
\hline S03_16707839 & qi03-1 & 3 & 16707839 & 0.17 & 7.12E-05 & 0.14 & 72 \\
\hline S03_16748994 & & 3 & 16748994 & 0.61 & $3.33 \mathrm{E}-06$ & 0.03 & 74 \\
\hline S03_16777244 & & 3 & 16777244 & 0.87 & 3.73E-06 & 0.02 & 61 \\
\hline S03_16789846 & & 3 & 16789846 & 0.66 & $1.90 \mathrm{E}-05$ & 0.08 & 59 \\
\hline S06_10959696 & qi06-1 & 6 & 10959696 & 0.75 & $6.81 \mathrm{E}-05$ & 0.16 & 7 \\
\hline S07_18744029 & qi07-1 & 7 & 18744029 & 0.45 & $6.49 E-04$ & & 24 \\
\hline S07_18908531 & qi07-2 & 7 & 18908531 & 0.53 & $9.17 E-04$ & & 17 \\
\hline S08_24524064 & qi08-1 & 8 & 24524064 & 0.54 & $3.25 \mathrm{E}-05$ & 0.11 & 38 \\
\hline S08_24620154 & qi08-2 & 8 & 24620154 & 0.51 & $1.02 E-04$ & & 36 \\
\hline S09_12474280 & qi09-1 & 9 & 12474280 & 0.83 & $5.65 \mathrm{E}-07$ & 0.01 & 33 \\
\hline S09_12772588 & qi09-2 & 9 & 12772588 & 0.10 & 5.85E-05 & 0.16 & 30 \\
\hline S11_28821256 & qi11-1 & 11 & 28821256 & 0.59 & $1.06 E-03$ & & 14 \\
\hline
\end{tabular}

In bold, markers significant in the initial analysis; in italics, initial probability of the markers being significant through sub-sampling

(posterior probabilities of 0.55 to 0.86 ), and five new significant markers were added (Table 5). By comparison with the indica panel, the significant markers all had a low to very low MAF. We determined which lines had the low-frequency alleles at these significant markers. The lines involved were different from marker to marker and generally included accessions with extremes phenotypes (from subpopulations J4 or J6) associated with one or several accessions with rather average phenotypes from the same or other subpopulations.

A GWAS was then conducted on the japonica panel with the HDRA dataset (Table 6). The same phenomenon was observed as with the GBS data but was more pronounced: long stretches of markers in complete LD on chromosome segments and markers in complete LD located on different chromosomes (up to 828 markers in one case). The analysis enabled the identification of 19 markers $(p<5 \mathrm{e}-06)$ corresponding to 18
QTLs. Three of these QTLs (qj01-1 on chr 1, qj07-2 on chr 2 and qj11_2 on chr11) corresponded to QTLs identified using the GBS data. As with the GBS data, all the detected QTLs had a very low MAF.

To assess the extent to which these results were influenced by the group of 10 accessions with a very wide root cone angle, we repeated the same analyses on the panel excluding those 10. This time, the model considering kinship and structure accurately controlled the number of false positives (Additional file 10: Figure S7). No marker was significant at $p<1-04$, probably because of the drastic reduction in the range of phenotypic values. The sub-sampling, with a threshold of 0.16 this time, identified six markers and five QTLs that all corresponded to the markers significant at $\mathrm{p}<5$-e04 in the initial analyses (Table 7; Additional file 11: Figure S8). None of these markers had a low or very low MAF. These results showed that the exclusion of the 10 outlier

Table 4 Markers significant in the HDRA indica panel

\begin{tabular}{|c|c|c|c|c|c|c|}
\hline Marker & QTL & Chr & Position & $\mathrm{p}$-value & q-value & $\begin{array}{l}\text { Number obs } \\
\text { minor allele }\end{array}$ \\
\hline S01_25647740 & qi01-1 & 1 & 25647740 & $3.92 \mathrm{E}-06$ & & 37 \\
\hline S01_25673948 & & 1 & 25673948 & $2.92 \mathrm{E}-06$ & & 37 \\
\hline S02_11448899 & qi02-1 & 2 & 11448899 & 1.59E-06 & & 7 \\
\hline S04_19229271 & qi04-1 & 4 & 19229271 & $1.75 \mathrm{E}-06$ & & 5 \\
\hline S07_18909031 & qi07-2 & 7 & 18909031 & $1.55 \mathrm{E}-06$ & & 14 \\
\hline S07_22105379 & qi07-3 & 7 & 22105379 & $2.66 \mathrm{E}-06$ & & 6 \\
\hline S11_06103683 & qi11-1 & 11 & 6103683 & $1.08 \mathrm{E}-07$ & & 9 \\
\hline S12_22205736 & qi12-1 & 12 & 22205736 & $1.72 \mathrm{E}-07$ & & 5 \\
\hline S12_22231768 & & 12 & 22231768 & 9.82E-07 & & 14 \\
\hline
\end{tabular}

In bold, markers or intervals in common with GBS ones 
Table 5 Markers significant in the GBS japonica panel

\begin{tabular}{|c|c|c|c|c|c|c|c|c|}
\hline Marker & QTL & Chr & Position1 & Position2 & Posterior probability & $p$-value & q-value & $\begin{array}{l}\text { Number obs. } \\
\text { minor allele }\end{array}$ \\
\hline S01_23183181 & qj01-1 & 1 & 23183181 & 23351413 & 0.65 & $3.76 \mathrm{E}-05$ & 0.07 & 6 \\
\hline S01_27104121 & qj01-2 & 1 & 27104121 & 27146133 & 0.55 & $8.36 \mathrm{E}-05$ & 0.11 & 8 \\
\hline S02_14095794 & qj02-1 & 2 & 14095794 & & 0.54 & $2.31 E-04$ & & 6 \\
\hline S02_32333192 & qj02-2 & 2 & 32333192 & 32457973 & 0.57 & $1.89 E-04$ & & 6 \\
\hline S05_21144094 & qj05-1 & 5 & 21144094 & & 0.01 & $3.15 \mathrm{E}-06$ & 0.03 & 5 \\
\hline S05_24706117 & qj05-2 & 5 & 24706117 & 24734678 & 0.01 & $1.33 \mathrm{E}-05$ & 0.05 & 5 \\
\hline S06_00185125 & qj06-1 & 6 & 185125 & & 0.01 & 4.06E-05 & 0.07 & 8 \\
\hline S06_21685315 & qj06-2 & 6 & 21685315 & & 0.71 & $3.03 \mathrm{E}-05$ & 0.07 & 14 \\
\hline S06_28055840 & qj06-3 & 6 & 28055840 & & 0.77 & $2.51 \mathrm{E}-05$ & 0.07 & 13 \\
\hline S07_15566174 & qj07-1 & 7 & 15566174 & & 0.71 & $8.22 \mathrm{E}-05$ & 0.12 & 6 \\
\hline S07_27048508 & qj07-2 & 7 & 27048508 & 27051011 & 0.59 & $9.12 \mathrm{E}-05$ & 0.11 & 9 \\
\hline S07_27060189 & & 7 & 27060189 & & 0.66 & 4.61E-05 & 0.07 & 8 \\
\hline S08_03918947 & qj08-1 & 8 & 3918947 & & 0.86 & $2.53 \mathrm{E}-06$ & 0.04 & 12 \\
\hline S09_17835213 & qj09-1 & 9 & 17835213 & & 0.54 & $1.55 E-04$ & & 16 \\
\hline S10_23032257 & qj10-1 & 10 & 23032257 & & 0.01 & $9.02 \mathrm{E}-06$ & 0.05 & 12 \\
\hline S11_03638312 & qj11-1 & 11 & 3638312 & 3667586 & 0.64 & $1.68 E-04$ & & 8 \\
\hline S11_23650929 & qj11-2 & 11 & 23650929 & & 0.73 & 2.41E-05 & 0.08 & 5 \\
\hline
\end{tabular}

In bold, marker significant in the intial analysis; in italic initial probability of the markers only significant through sub-sampling. Position1-position 2: limits of intervals including significant markers in complete LD

Table 6 Markers significant in the HDRA japonica panel

\begin{tabular}{|c|c|c|c|c|c|c|c|}
\hline Marker & QTL & Chr & Position 1 & Position 2 & $p$-value & q-value & $\begin{array}{l}\text { Number obs. } \\
\text { minor allele }\end{array}$ \\
\hline S01_01196103 & qj01-3 & 1 & 1196103 & & $9.68 \mathrm{E}-07$ & 0.04 & 14 \\
\hline S01_22981270 & qj01-1 & 1 & 22981270 & 23571718 & $1.29 \mathrm{E}-06$ & 0.04 & 5 \\
\hline S01_27982591 & qj01-4 & 1 & 27982591 & & 2.30E-06 & 0.05 & 8 \\
\hline S01_41631488 & qj01-5 & 1 & 41631488 & & 4.07E-06 & 0.06 & 9 \\
\hline S02_24998179 & qj02-3 & 2 & 24998179 & & $3.04 \mathrm{E}-06$ & 0.07 & 5 \\
\hline S03_22066093 & qj03-1 & 3 & 22066093 & & $6.27 \mathrm{E}-10$ & $<0.01$ & 9 \\
\hline S04_30759181 & qj04-1 & 4 & 30759181 & & $1.77 \mathrm{E}-06$ & 0.04 & 11 \\
\hline S05_04237081 & qj05-3 & 5 & 4237081 & 4240625 & $3.17 \mathrm{E}-06$ & 0.06 & 5 \\
\hline S05_06615188 & qj05-4 & 5 & 6615188 & & $7.55 \mathrm{E}-07$ & 0.05 & 5 \\
\hline S06_20926166 & qj06-4 & 6 & 20926166 & & 1.10E-07 & 0.02 & 5 \\
\hline S07_27039086 & qj07-2 & 7 & 27039086 & 27067119 & $5.72 \mathrm{E}-07$ & 0.04 & 6 \\
\hline S11_21915814 & qj11-3 & 11 & 21915814 & 21986377 & $1.28 \mathrm{E}-06$ & 0.04 & 5 \\
\hline S11_23438923 & qj11-4 & 11 & 23438923 & & 4.00E-06 & 0.07 & 6 \\
\hline S11_23640395 & qj11-2 & 11 & 23640395 & & $1.41 \mathrm{E}-06$ & 0.04 & 5 \\
\hline S11_24364407 & qj11-5 & 11 & 24364407 & & $1.28 \mathrm{E}-06$ & 0.04 & 6 \\
\hline S11_24388283 & & 11 & 24388283 & & $9.43 \mathrm{E}-07$ & 0.05 & 7 \\
\hline S11_25610599 & qj11-6 & 11 & 25610599 & & $1.43 \mathrm{E}-07$ & 0.01 & 8 \\
\hline S12_17977193 & qj12-1 & 12 & 17977193 & & $4.05 \mathrm{E}-06$ & 0.07 & 12 \\
\hline S12_24921245 & qj12-2 & 12 & 24921245 & & $3.16 \mathrm{E}-06$ & 0.06 & 6 \\
\hline
\end{tabular}

In bold, markers or intervals common with GBS ones 
Table 7 Markers significant in the GBS japonica panel excluding the 10 extreme lines with very wide root cone angles

\begin{tabular}{|c|c|c|c|c|c|c|c|}
\hline Marker & QTL & Chr & Position1 & Position2 & Posterior probability & $p$-value & $\begin{array}{l}\text { Number obs } \\
\text { minor allele }\end{array}$ \\
\hline S01_39102231 & qj01-6 & 1 & 39102231 & & 0.19 & $4.75 E-04$ & 34 \\
\hline S01_41207248 & qj01-7 & 1 & 41207248 & 41304330 & 0.30 & $2.08 E-04$ & 17 \\
\hline S01_41249221 & & 1 & 41249221 & & 0.40 & $1.42 E-04$ & 18 \\
\hline S04_31661682 & qj04-2 & 4 & 31661682 & & 0.21 & 4.56E-04 & 62 \\
\hline S05_04482655 & qj05-5 & 5 & 4482655 & & 0.31 & $2.88 E-04$ & 29 \\
\hline S11_20481199 & qj11-7 & 11 & 20481199 & & 0.16 & 4.26E-04 & 61 \\
\hline
\end{tabular}

In italics, initial probability of the markers only significant through sub-sampling. Position 1-position 2: limits of intervals including significant markers in complete LD

accessions allowed the targeting of another set of genes involved in root cone angles. A GWAS was then conducted on the reduced japonica panel with the HDRA dataset (Table 8). Four markers on chr 2, 3, 7, and 12 were significant but had a relatively high qvalue (from 0.17 to 0.36 ). None of these markers were shared with those detected in the GBS dataset.

To identify the loci responsible for the very wide root cone angle, assuming that this trait can be controlled by a major gene, we tested other approaches using only the GBS dataset since phenotypic and genotypic data were available for 10 of the extreme accessions compared with only 8 in the HDRA dataset. First, we looked at markers that were discriminant between the 10 accessions with an open root cone angle and the rest of the panel (157 accessions). Marker S06_21,685,315, corresponding to qj06_02 was found to be the one that discriminated the best the two phenotypic groups. The variant allele present in the 10 accessions with a wide root cone angle was present in only four accessions of the rest of the panel. However, these four accessions included Ketan Lumbu (bulu) and Padi Kasale (close to bulus), which had wide root cone angles (close to $100^{\circ}$ ), as well as NPE253 and NPE826 (temperate japonica) from Pakistan, which had narrow angles (approximately 50 $)$. In contrast, when the reference allele was present in the 10 accessions, no marker was found discriminant with at best a ratio of 7 accessions with the reference allele compared with 150 accessions with the variant allele in the rest of the panel.

These results led us to assume that the wide root cone angle trait could be controlled by different mutations in the J4 and J6 subpopulations. It was not possible to test this hypothesis with $\mathrm{J} 4$ since all alleles specific to the subpopulation would have appeared associated with the mutation. However, it was possible to avoid structural problems with J6, since J6 was composed of only accessions of the two tails of the distribution. The same approach as above was therefore applied to J6. The allele frequency among the three accessions of J6 with open root angles was compared to the allele frequency among the 7 accessions with narrow root angles. Because of the very small size of the sample, only the extreme situation (one allele present in one group and absent in the other or vice versa) was considered. This time, 367 and 132 markers distributed in cluster on different chromosomes were found discriminant within J6 for the variant and the reference alleles, respectively. However, all these markers segregated in the rest of the population, with at best a ratio of 2 accessions with the reference or variant alleles compared with 155 with the complementary one.

We ended with 15 QTLs for the indica panel and 40 QTLs for the japonica panel (Fig. 2). None of the significant markers were located in similar zones in the indica and japonica panels.

\section{Analysis of the candidate genes}

The positions of these 55 QTLs were compared to the position of root-related genes in rice from the EURoot database. No known root gene corresponded to the markers that were found to be significant in the indica panel. For the japonica panel, five genes present in the EURoot database co-localized with significant markers: SHORT POSTEMBRYONIC ROOTS 1 (OsSPR1 / Os0

Table 8 Markers significant in the HDRA japonica panel excluding the 8 extreme lines with very wide root cone angle

\begin{tabular}{|c|c|c|c|c|c|c|}
\hline Marker & QTL & Chr & Position & $p$-value & q-value & $\begin{array}{l}\text { Number obs. } \\
\text { minor allele }\end{array}$ \\
\hline S02_25551591 & qj02-4 & 2 & 25551591 & $5.71 \mathrm{E}-07$ & 0.17 & 9 \\
\hline S03_22524470 & qj03-2 & 3 & 22524470 & $4.76 \mathrm{E}-06$ & 0.36 & 5 \\
\hline S07_00929748 & qj07-3 & 7 & 929748 & 2.81E-06 & 0.28 & 5 \\
\hline S12_15655309 & qj12-3 & 12 & 15655309 & $2.40 \mathrm{E}-06$ & 0.36 & 21 \\
\hline
\end{tabular}


1g67290) $43.3 \mathrm{~kb}$ from qj01_6, CYTOKININ OXIDASE/ DEHYDROGENASE 4 (OsCKX4 / Os01g71310) within qj01_7, PHOSPHATE TRANSPORTER 15 (OsPT15 / Os0 2g52860) 9.9 kb from qj02_2, NOD26-LIKE INTRINSIC P 1;4 (OsNIP1;4 / Os06g35930) 34. $5 \mathrm{~kb}$ from qj06-4 and CELLULOSE SYNTHASE-LIKE FAMILY D (OSCSLD1 / Os10g42750) $30.2 \mathrm{~kb}$ from qj10_1.

Finally, the genes included in an interval of $+/-50 \mathrm{~kb}$ around the markers were analyzed. We found 489 genes with an annotated function, among which 92 were localized within a QTL, and 91 were located in a 10-kb zone around a significant marker (Additional file 12: Table S4). Among these genes, based on Michigan State University annotations (http://rice.plantbiology.msu.edu/), 29 genes were kinases, including 6 LRR-RLKs, and 5 genes were linked to the auxin pathway (three $3 \mathrm{~F}$-box domain and LRR- containing proteins and two members of the auxin-responsive SAUR gene family). The 17 genes for which a link with roots could be found either in rice or through orthology with Arabidopsis are summarized in Table 9.

\section{Discussion}

We ran GWASs on root cone angle in indica and japonica panels of intermediate size, which were genotyped with two large sets of markers. The root cone angle was assessed in a total population of 331 accessions and enabled the identification of accessions with exceptionally wide root cone angles. This phenotype seems relatively frequent in O. sativa and distributed in different varietal groups. In our japonica panel, these accessions included all those belonging to the bulu ecotype, confirming the results obtained by Ueno and Sato $(1989,1992)$ and a few temperate japonica lines from South Asia that had not yet been identified as carrying such mutations. Ueno and Sato (1992) also found accessions with wide root cone angle among the aus and boro ecotypes from India and tjereh from Indonesia that belong to the indica subspecies. We did not find any such line in our indica panel, but these specific ecotypes were represented by very few accessions, probably too few to detect the mutation. Not considering mutants, the root cone angles of the indica and japonica panels largely overlapped, while indica accessions are said to be generally shallow rooted and japonica accessions to be deep rooted (Lafitte et al. 2001). One could wonder whether this result is specific to our phenotyping system. However, Uga et al. (2009), using the 3D basket system, also concluded that there was no significant difference in the distribution of an index based on root cone angle between the indica and japonica groups. On an accession basis, Clark et al. (2011), using a gellan gum culture system, did not find differences in root initiation angle between IR64 and Azucena, our two controls in the present study that are nonetheless known to have contrasting root depths (Yadav et al. 1997). These results confirm those from Abe and Morita (1994) that the link between narrow root cone angle and increased root depth cannot be generalized in rice.

Since our panels had been genotyped by two different methods (GBS and high-density chip), we assessed whether the number of markers was a limitation for association detection by comparing the results obtained with the GBS data sets (approximately 15,000 markers) and the HDRA datasets (approximately 300,000 markers). Theoretically, the large increase in marker density should not lead to the identification of many more associations because, in both the indica and the japonica panels, LD decay is much slower than the average distance between markers even in the two GBS datasets (Courtois et al. 2013; Lafarge et al. 2017). However, this statement is based on averages, and locally, large variations or ruptures in LD pattern can be observed, because of recent mutations in some lineages, for example. In such cases, the HDRA dataset has a greater chance of showing a higher LD between the causative polymorphism and the genotyped markers than does the GBS dataset. Based on our data, the HDRA dataset associations recorded much lower $p$-values than did those from the GBS dataset (minimum $p$-value of $6.3 \mathrm{e}-10$ for the HDRA compared with 2.5 e-06 for the GBS), but when using a threshold that considered the difference in the number of markers between datasets, little difference in the number of significant associations was observed due to the 20-fold-increase in marker density between the GBS and HDRA datasets. However, one cannot exclude that this lack of difference results partly from the panel sizes, which are smaller for the HDRA datasets. Improvement in resolution can be obtained by an increase in panel size. In our case, this may have been achieved by pooling the indica and japonica panels. We did not attempt to do so since the conditions for pooling the datasets were not optimal. The experiments with the two panels were conducted independently, and the common controls (IR64 and Azucena) that could have enabled us to bridge the phenotypic datasets registered similar values for root cone angle, making them poor controls for this trait although showing major differences in other root traits.

For both panels, the significant markers in the GBS and HDRA datasets pinpointed to similar chromosomal segments in several cases but there were also chromosomal segments where the markers were significant for one dataset and not for the other. One possible reason, besides the difference in panel sizes mentioned above, is the fact that the two datasets were submitted to different ascertainment biases in SNP selection. The HDRA dataset, derived from sequence data, is enriched in SNPs in and around genes (McCouch et al. 2016) while the 
Table 9 List of the most interesting genes underlying the QTLS

\begin{tabular}{|c|c|c|c|c|c|c|c|c|}
\hline Gene & Chr & $\begin{array}{l}\text { Start } \\
\text { position }\end{array}$ & $\begin{array}{l}\text { Stop } \\
\text { position }\end{array}$ & QTL & Distance from mk (in kb) & Kinase & $\begin{array}{l}\text { Kinase } \\
\text { sub-group }\end{array}$ & Annotation (MSU) \\
\hline Os01g41340 & 1 & 23392955 & 23394611 & qj01-1 & IN & No & & $\begin{array}{l}\text { OsFBL1; F-box domain- and LRR- } \\
\text { containing protein }\end{array}$ \\
\hline Os01g41530 & 1 & 23510098 & 23512343 & qj01-1 & IN & No & & $\begin{array}{l}\text { OsFBL2; F-box domain- and LRR- } \\
\text { containing protein }\end{array}$ \\
\hline Os01g48850 & 1 & 28028738 & 28032031 & qj01-4 & 46.1 & No & & Auxin-responsive protein putative \\
\hline Os01g67290 & 1 & 39052262 & 39058974 & qj01-6 & 43.3 & No & & OsSPR1; cyclin-related protein putative \\
\hline Os01g67340 & 1 & 39091728 & 39095339 & qj01-6 & 6.9 & Yes & RLCK-VIII & $\begin{array}{l}\text { STRUBBELIG-RECEPTOR FAMILY } 8 \\
\text { precursor putative }\end{array}$ \\
\hline Os01g71310 & 1 & 41300203 & 41303044 & qj01-7 & IN & No & & $\begin{array}{l}\text { OsCKX4; cytokinin dehydrogenase } \\
\text { precursor }\end{array}$ \\
\hline Os02g41650 & 2 & 24985255 & 24989388 & qj02-3 & 8.8 & No & & $\begin{array}{l}\text { Phenylalanine ammonia-lyase protein } \\
\text { (PAL) putative }\end{array}$ \\
\hline Os02g52860 & 2 & 32319364 & 32323332 & qj02-2 & 9.9 & No & & $\begin{array}{l}\text { OsPT15; phosphate carrier protein } \\
\text { mitochondrial } \\
\text { precursor }\end{array}$ \\
\hline Os02g52990 & 2 & 32418493 & 32419361 & qj02_2 & IN & No & & $\begin{array}{l}\text { OSSAUR12; auxin-responsive SAUR } \\
\text { gene family member }\end{array}$ \\
\hline Os04g51890 & 4 & 30783636 & 30784530 & qj04-1 & 24.4 & No & & $\begin{array}{l}\text { OsSAUR20; auxin-responsive SAUR } \\
\text { gene family member }\end{array}$ \\
\hline Os05g11730 & 5 & 6657481 & 6661493 & qj05-4 & 42.3 & Yes & GSK2 & $\begin{array}{l}\text { CGMC_GSK.7; CGMC includes CDA, } \\
\text { MAPK, GSK3, and CLKC kinases }\end{array}$ \\
\hline Os06g35930 & 6 & 20960706 & 20961819 & qj06-4 & 34.5 & No & & NIP1;4; aquaporin protein, putative \\
\hline Os07g36900 & 7 & 22095130 & 22099277 & qi07-3 & 6.1 & No & & $\begin{array}{l}\text { OsFBL39; F-box domain and LRR- } \\
\text { containing protein }\end{array}$ \\
\hline Os09g20740 & 9 & 12494569 & 12498572 & qi09-1 & 20.3 & Yes & WAK & $\begin{array}{l}\text { OsWAK79; OsWAK receptor-like } \\
\text { protein kinase }\end{array}$ \\
\hline Os10g42750 & 10 & 23062454 & 23066292 & qj10-1 & 30.2 & No & & $\begin{array}{l}\text { OsCSLD1; cellulose synthase-like } \\
\text { family D }\end{array}$ \\
\hline Os11g07230 & 11 & 3633303 & 3638663 & qj11-1 & IN & Yes & LRR-XII & Receptor kinase, putative \\
\hline Os11g07240 & 11 & 3640868 & 3644003 & qj11-1 & IN & LRR-RLK & SG_XIla-6c & $\begin{array}{l}\text { Serine/threonine-protein kinase } \\
\text { BRI1-like } 2 \text { precursor, } \\
\text { putative }\end{array}$ \\
\hline Os11g39370 & 11 & 23431233 & 23436807 & qj11-4 & 2.1 & Yes & OSSERL 1 & $\begin{array}{l}\text { BRASSINOSTEROID INSENSITIVE 1- } \\
\text { aSSociated receptor kinase } 1 \text { precursor }\end{array}$ \\
\hline
\end{tabular}

markers of the GBS dataset were more randomly selected. The proportion of markers with a low MAF of the HDRA dataset was also much higher than that of the GBS dataset $(63 \%$ and $69 \%$ of markers with MAF $<10 \%$ for the indica and japonica HDRA panels compared with 38.0 and $46.2 \%$ for the indica and japonica GBS panels, respectively) although the same filtering procedure was used. Better balanced genotypic classes, as in the GBS dataset, generally provide higher power for association detection.

We detected significant associations and tested the robustness of those associations using a resampling technique. Resampling enables the evaluation of the sensitivity of the tests to the specificities of the samples (Tian et al. 2011). Some of the QTLs were much better supported than others. While we did not encounter methodological difficulties in conducting GWAS in the indica panel, we did in the japonica panel. The root cone angle in the japonica panel is a clear example of a trait for which GWAS may not be the best way to quickly identify the underlying gene(s). Two problems surface. The first problem comes from the fact that one subpopulation, J4, consisting of accessions from the bulu ecotype, is composed of only one-tail extremes. In such case, correcting population structure will remove the significant associations. The second problem is that, in subpopulation J6, the number of extreme accessions is very low (only 3 accessions) and GWAS is not appropriate for analyzing situations with rare alleles (Gibson 2012; Zhang et al. 2016). In addition, as noted by Korte and Farlow (2013), rare alleles that are specific to such small sub-groups of individuals will be in complete LD, which is what we observed with markers with the same $p$-value on different chromosomes and drove us to 
eliminate many significant markers. Reverting to classical mapping populations such as RILs deriving from two divergent parents will solve these problems of the confounding effect of population structure and low frequency alleles. The poorer resolution, which is generally registered in classical mapping populations in comparison with GWAS panels, can easily be resolved by increasing the population size. Such a population was developed by Uga et al. (2012) who crossed a temperate japonica with a narrow root cone angle with a bulu with an open root cone angle. A mapping population involving one extreme parent of subpopulation $\mathrm{J} 4$ and one from J6 is another option if the objective is to determine whether the phenotypes observed in the various subpopulations are allelic.

Several root-related genes were identified under or close to the significant markers. We screened the literature to determine the biological function of these genes in rice or that of their orthologs in Arabidopsis when no other information was available. First, five genes were found in the EURoot database. Three of them were described as involved in root nutrient transport, in water transport and in root hairs. OsPT15 (Os02g52860) codes for a mitochondrial phosphate transporter (Liu et al. 2011). OsNIP1;4 (Os06g35930) is an aquaporin that is expressed in leaf blades and in roots involved in water transport in rice (Sakurai et al. 2008). OsCSLD1 (Os10g42750) is implicated in the growth of the primary cell wall at the root hair tip. The two other genes are described as affecting root architecture. OSSPR1 codes for a mitochondrial protein involved in the maintenance of Fe homeostasis. An OSSPR1 mutant has deficient root elongation in primary, adventitious and lateral roots due to the reduction in root cell length (Jia et al. 2011). OsCKX4, which codes for an enzyme responsible for cytokinin degradation, positively regulates crown root initiation and development in rice (Gao et al. 2014). This gene possesses an auxin response element in its promoter, which is the target of the auxin-response factor OsARF25. Auxin induces OsCKX4 gene expression in roots. Knock-out lines of OsCKX4 show an inhibition of the primary roots and fewer crown roots at the seedling stage. Conversely, lines over-expressing OsCKX4 show enhanced root system development.

We also identified five genes related to the auxin signaling pathway and auxin response. Three of these genes belong to the F-box-domain- and LRR-containing protein family, but no biological function has been assigned to the three specific FBLs found in this study. The FBL family, which is a subdivision of the large F-Box family, includes only 61 members (Jain et al. 2007). Only a few of them (OsFLB16 (AFB2), OsFLB21 (TIR1) and $D W A R K 3$ (OsFLB27)) have been biologically studied. All were shown to have an impact on root architecture, notably on crown and lateral root development. OsFBL16 and OsFBL21 interact with OsIAA1 and alter auxin signaling (Bian et al. 2012). OsSAUR12 and OsSAUR2O belong to an auxin responsive family of 58 genes in rice. They both encode a protein bearing an auxin-inducible domain of $118 \mathrm{AA}$, but its biological function has not yet been determined in rice. However, OsSAUR12 is down-regulated in the mutant of the CROWN ROOTLESS 1 (CRL1) gene (Coudert et al. 2011). CRL1 is involved in crown root development and is induced by auxin (Inukai et al. 2005). The last auxin-related gene identified, Os01g48850, encodes a putative auxin-responsive protein that has not been studied in rice, but its ortholog in Arabidopsis, At3g61750, is member of the family of DOMONcontaining proteins, of which some members are involved in root development (Preger et al. 2009).

Several kinases have an effect on root gravitropism responses (reviewed by Armengot et al. (2016)). In our study, we found 29 kinases belonging to different subfamilies. We identified six $L R R-R L K s$, two $L-L E C T I N$ kinases, three $R E$ CEPTOR-LIKE CYTOPLASMIC KINASES (RLCKS), one $W A K$, and a few other kinases belonging to different subgroups (Additional file 12 Table S4), but no biological function had been assigned to most of these genes in either rice or Arabidopsis. Among the LRR-RLKs with biological function, Os11g07240 (SG_XIIa), which belongs to a cluster of 5 LRR-RLK genes on chr11, has been studied by Chen et al. (2013). Os11g07240, called HYBRID WEAKNESS 1 (Hw1), is proposed to be responsible for autoimmunity activation leading to a hybrid weakness phenotype. A nearisogenic line (NIL) carrying an O. rufipogon allele at this gene in an elite variety background displays defaults in root development and notably fewer crown roots due to a drastic diminution of crown root primordia. Os11g39370 encodes a protein annotated BRASSINOSTEROID INSENSITIVE 1-associated receptor kinase 1 precursor and belongs to the SERK family (LRR-RLK SG_II). It is classified in the same SG as DOCS1, which is known to affect root cone angle (Bettembourg 2016), but to date, no function has been assigned to this gene. Among the other LRR$R L K s$, Os01g67340 codes for a protein annotated STRUBBELIG-RECEPTOR FAMILY protein that has not yet been studied in rice. In Arabidopsis, mutants of a member of the same family, SCRAMBLED $(S C M)$, are affected in root hair specification (Kwak and Schiefelbein 2007).

Lastly, Os02g41650, which is part of a cluster of three genes with similar biochemical function, codes for a putative phenylalanine ammonia-lyase (PAL) protein and has recently been studied in relation to gravitropism $(\mathrm{Hu}$ et al. 2013). It was hypothesized that this gene contributed to the synthesis of kaempferol and myricetin (two 
flavonols) in shoot tissues and regulated the transport of auxin during gravitropism.

\section{Conclusions}

The root cone angle is an important trait because it largely determines the volume of soil that a plant explores. In the present study, we identified new sources of large root cone angle in a temperate japonica subpopulation in addition to those already known from the bulu ecotype. We encountered difficulties in using GWAS for this trait in japonica rice and proposed other approaches for further genetic analyses. From candidate gene analysis, we identified eight genes close to significant markers (OsSAUR12 in qj02-2, OsCKX4 in qj01-7, OsSPR1 near qj01-6, OsCSLD1 near qj10-1, STRUBBELIG-RECEPTOR FAMILY 8 near qj01-6, BRASSINOTEROID INSENSITIVE 1 near qj11-4, Hw1 near qj11-1 and $P A L$ near qj02-3) that seem to be candidates deserving further investigation.

\section{Additional files} Additional file 1: Table S1. List of QTLs and genes for root growth
angle and related traits (XLSX $16 \mathrm{~kb})$

Additional file 2: Table S2. List of the accessions included the indica panel with their phenotype. (XLSX $25 \mathrm{~kb}$ )

Additional file 3: Table S3. List of the accessions included the japonica panel with their phenotype. (XLSX $27 \mathrm{~kb}$ )

Additional file 4: Figure S1. Neighbor joining tree of the indica panel. The colors corresponds to the subpopulations defined by Structure. The accessions in black are admixed (PPTX $163 \mathrm{~kb}$ )

Additional file 5: Figure S2. Neighbor joining tree of the japonica panel. The colors corresponds to the subpopulations defined by Structure. The accessions in black are admixed (PPTX $158 \mathrm{~kb}$ )

Additional file 6: Figure S3. Q-q plot for root cone angle in the indica panel with GBS data. (JPEG $68 \mathrm{~kb}$ )

Additional file 7: Figure S4. Manhattan plot for the indica panel with GBS data. P-values by chromosome for root cone angle. (JPEG 98 kb)

Additional file 8: Figure S5. Q-q plot for root cone angle in the japonica panel with GBS data. (JPEG $73 \mathrm{~kb}$ )

Additional file 9: Figure S6. Manhattan plot for the japonica panel with GBS data. P-values by chromosome for root cone angle. (JPEG 142 kb)

Additional file 10: Figure S7. Q-q plot for root cone angle for the japonica panel, 10 extreme lines excluded, with GBS data. (JPEG 67 kb)

Additional file 11: Figure S8. Manhattan plot for the japonica panel, 10 extreme lines excluded, with GBS data. P-values by chromosome for root cone angle. (JPEG $131 \mathrm{~kb}$ )

Additional file 12: Table S4. List of genes with annotated function underlying the QTLs (XLSX 47 kb)

\section{Acknowledgements}

This research received funding from the International Rice Research Institute in the framework of the Rhizophen project for the experiments conducted with the indica panel. This research received funding from the European Community Seventh Framework Programme FP7-KBBE-2011-5 under grant agreement no 289300 in the framework of EURoot project for the experiment with the japonica panel. The authors thank all the Cirad staff who contributed to the various experiments. The authors thank Chantal Hamelin, from Cirad, for making the genotypic data available in the Tropgene database.

\section{Availability of data and materials}

All four genotypic data under HapMap format (indica and japonica GBS and indica and japonica HDRA) are available for download at http://tropgenedb.cirad.fr/ tropgene/JSP/interface.jsp?module=RICE under study type "genotypes" as a zip file named "Root cone angle". The phenotypic data are included in Tables S2 and S3 in Excel format.

\section{Authors' contributions}

$M B, A D, E G$ and $B C$ designed the experiment. $A A, A D a, E T$ and $B C$ phenotyped the panels. JF prepared the GBS and HDRA final datasets. NA and $B C$ defined the GWAS analysis methodology. $M B$ and $B C$ performed the GWAS analysis. MB, AD and CP analyzed the candidate genes. $M B, B C$ and AD wrote the paper, which was edited and approved by all co-authors.

\section{Competing interests}

The authors declare that they have no competing interests.

\section{Publisher's Note}

Springer Nature remains neutral with regard to jurisdictional claims in published maps and institutional affiliations.

\section{Author details}

${ }^{1}$ Cirad, UMR AGAP, F34398 Montpellier Cedex 5, France. ${ }^{2}$ Cirad / ISRA-Ceraas, BP 3320 Thies, Senegal. ${ }^{3}$ Shanghai Jiao Tong University (SJTU), School of Life Sciences and Biotechnology, Shanghai 200240, China.

Received: 29 June 2017 Accepted: 19 September 2017

Published online: 02 October 2017

\section{References}

Abe J, Morita S (1994) Growth direction of nodal roots in rice: its variation and contribution to root system formation. Plant Soil 165:333-337

Ahmadi N, Audebert A, Bennett MJ, Bishopp A, de Oliveira AC, Courtois B, Diedhiou A, Dievart A, Gantet P, Ghesquiere A, Guiderdoni E, Henry A, Inukai Y, Kochian L, Laplaze L, Lucas M, Luu DT, Manneh B, Mo XR, Muthurajan R, Perin C, Price A, Robin S, Sentenac $H$, Sine B, Uga Y, Very AA, Wissuwa M, Wu $P, X u J ~(2014)$ The roots of future rice harvests. Rice 7:29-38

Alexandrov N, Tai S, Wang W, Mansueto L, Palis K, Fuentes RR, Ulat VJ, Chebotarov D, Zhang G, Li Z (2015) SNP-Seek database of SNPs derived from 3000 rice genomes. Nucleic Acids Res 43(d1):1023-1027

Armengot L, Marquès-Bueno MM, Jaillais Y (2016) Regulation of polar auxin transport by protein and lipid kinases. J Exp Bot 67(14):4015-4037

Bettembourg M (2016) Caractérisation de récepteurs à activité kinase impliqués dans la mise en place de l'architecture racinaire chez le riz. PhD thesis, University of Montpellier-SupAgro, France, p 275

Bian H, Xie Y, Guo F, Han N, Ma S, Zeng Z, Wang J, Yang Y, Zhu M (2012) Distinctive expression patterns and roles of the miRNA393/TIR1 homolog module in regulating flag leaf inclination and primary and crown root growth in rice (Oryza sativa). New Phytol 196(1):149-161

Bradbury PJ, Zhang Z, Kroon DE, Casstevens TM, Ramdoss Y, Buckler ES (2007) TASSEL: software for association mapping of complex traits in diverse samples. Bioinformatics 23:2633-2635

Browning SR, Browning BL (2007) Rapid and accurate haplotype phasing and missing-data inference for whole-genome association studies by use of localized haplotype clustering. Am J Human Genet 81(5):1084-1097

Chen C, Chen H, Shan J-X, Zhu M-Z, Shi M, Gao J-P, Lin H-X (2013) Genetic and physiological analysis of a novel type of interspecific hybrid weakness in rice. Mol Plant 6(3):716-728

Clark RT, MacCurdy RB, Jung JK, Shaff JE, McCouch SR, Aneshansley DJ, Kochian LV (2011) Three-dimensional root phenotyping with a novel imaging and software platform. Plant Physiol 156:455-465

Coudert Y, Bes M, Le Thi VA, Pre M, Guiderdoni E, Gantet P (2011) Transcript profiling of crown root less 1 stem base reveals new elements associated with crown root development in rice. BMC Genomics 12(1):387

Courtois B, Audebert A, Dardou A, Roques S, Ghneim-Herrera T, Droc G, Frouin J, Rouan L, Goze E, Kilian A, Ahmadi N, Dingkuhn M (2013) Genome-wide association mapping of root traits in a Japonica rice panel. PLoS One 8(11): e78037

Dievart A, Perin C, Hirsch J, Bettembourg M, Lanau N, Artus F, Bureau C, Noel N, Droc G, Peyramard M, Pereira S, Courtois B, Morel J-B, Guiderdoni E (2016) The phenome analysis of mutant alleles in leucine-rich repeat receptor-like 
kinase genes in rice reveals new potential targets for stress tolerant cereals. Plant Sci 242:240-249

Elshire RJ, Glaubitz JC, Sun Q, Poland JA, Kawamoto K, Buckler ES, Mitchell SE (2011) A robust, simple genotyping-by-sequencing (GBS) approach for high diversity species. PLoS One 6(5):e19379

Fischer I, Dievart A, Droc G, Dufayard JF, Chantret N (2016) Evolutionary dynamics of the leucine-rich repeat receptor-like kinase (LRR-RLK) subfamily in Angiosperms. Plant Physiol 170(3):1596-1610

Fukai S, Cooper M (1995) Development of drought-resistant cultivars using physiomorphological traits in rice. Field Crop Res 40(2):67-86

Gao S, Fang J, Xu F, Wang W, Sun X, Chu J, Cai B, Feng Y, Chu C (2014) CYTOKININ OXIDASE/DEHYDROGENASE4 integrates cytokinin and auxin signaling to control rice crown root formation. Plant Physiol 165(3):10351046

Gao X (2011) Multiple testing corrections for imputed SNPs. Genet Epidemiol 35(3):154-158

Garris AJ, Tai TH, Coburn J, Kresovich S, McCouch S (2005) Genetic structure and diversity in Oryza sativa L. Genetics 169(3):1631-1638

Gibson G (2012) Rare and common variants: twenty arguments. Nat Rev Genet 13(2):135-145

Gowda VRP, Henry A, Yamauchi A, Shashidhar HE, Serraj R (2011) Root biology and genetic improvement for drought avoidance in rice. Field Crop Res 122(1):1-13

Hanzawa E, Sasaki K, Nagai S, Obara M, Fukuta Y, Uga Y, Miyao A, Hirochika H, Higashitani A, Maekawa M, Sato T (2013) Isolation of a novel mutant gene for soil-surface rooting in rice (Oryza sativa L.) Rice 6(1):30-30

Hu L, Mei Z, Zang A, Chen H, Dou X, Jin J, Cai W (2013) Microarray analyses and comparisons of upper or lower flanks of rice shoot base preceding gravitropic bending. PLoS One 8(9):e74646

Huang X, Wei X, Sang T, Zhao Q, Feng Q, Zhao Y, Li C, Zhu C, Lu T, Zhang Z (2010) Genome-wide association studies of 14 agronomic traits in rice landraces. Nat Genet 42(11):961-967

Inukai Y, Sakamoto T, Ueguchi-Tanaka M, Shibata Y, Gomi K, Umemura I, Hasegawa Y, Ashikari M, Kitano H, Matsuoka M (2005) Crown rootless1, which is essential for crown root formation in rice, is a target of an AUXIN RESPONSE FACTOR in auxin signaling. Plant Cell 17(5):1387-1396

Jain M, Nijhawan A, Arora R, Agarwal P, Ray S, Sharma P, Kapoor S, Tyagi AK, Khurana JP (2007) F-Box proteins in Rice. Genome-wide analysis, classification, temporal and spatial gene expression during panicle and seed development, and regulation by light and abiotic stress. Plant Physiol 143(4):1467-1483

Jia L, Wu Z, Hao X, Carrie C, Zheng L, Whelan J, Wu Y, Wang S, Wu P, Mao C (2011) Identification of a novel mitochondrial protein, short postembryonic roots 1 (SPR1), involved in root development and iron homeostasis in Oryza sativa. New Phytol 189(3):843-855

Kato Y, Abe J, Kamoshita A, Yamagishi J (2006) Genotypic variation in root growth angle in rice (Oryza sativa L.) and its association with deep root development in upland fields with different water regimes. Plant Soil 287 $(1-2): 117-129$

Kitomi Y, Kanno N, Kawai S, Mizubayashi T, Fukuoka S, Uga Y (2015) QTLs underlying natural variation of root growth angle among rice cultivars with the same functional allele of DEEPER ROOTING 1. Rice 8(1):1-12

Korte A, Farlow A (2013) The advantages and limitations of trait analysis with GWAS: a review. Plant Methods 9(1):1

Kundur P, Ashwathanarayana R, Keerthi C, Brijesh A, Haris B, Sashidar H (2015) Genotypic variation for root growth orientation in rice under aerobic and well watered conditions. Int J of Current Research 7(5):16331-16336

Kwak S-H, Schiefelbein J (2007) The role of the SCRAMBLED receptor-like kinase in patterning the Arabidopsis root epidermis. Dev Biol 302(1):118-131

Lafarge T, Bueno C, Frouin J, Jacquin L, Courtois B, Ahmadi N (2017) Genomewide association analysis for heat tolerance at flowering detected a large set of genes involved in adaptation to thermal and other stresses. PLoS One 12(2):e0171254

Lafitte H, Champoux M, McLaren G, O'Toole J (2001) Rice root morphological traits are related to isozyme group and adaptation. Field Crop Research 71: $57-70$

Liu F, Chang X-J, Ye Y, Xie W-B, Wu P, Lian X-M (2011) Comprehensive sequence and whole-life-cycle expression profile analysis of the phosphate transporter gene family in rice. Mol Plant 4(6):1105-1122

Lou Q, Chen L, Mei H, Wei H, Feng F, Wang P, Xia H, Li T, Luo L (2015) Quantitative trait locus mapping of deep rooting by linkage and association analysis in rice. J Exp Bot 66(15):4749-4757
Lynch JP, Brown KM (2001) Topsoil foraging. An architectural adaptation of plants to low phosphorus availability. Plant Soil 237(2):225-237

McCouch SR, Wright MH, Tung C-W, Maron LG, McNally KL, Fitzgerald M, Singh N, DeClerck G, Agosto-Perez F, Korniliev P, Greenberg AJ, Naredo MEB, Mercado SMQ, Harrington SE, Shi Y, Branchini DA, Kuser-Falcao PR, Leung H, Ebana K, Yano M, Eizenga G, McClung A, Mezey J (2016) Open access resources for genome-wide association mapping in rice. Nat Commun 7:10532

Norton GJ, Price AH (2009) Mapping of quantitative trait loci for seminal root morphology and gravitropic response in rice. Euphytica 166:229-237

OToole J, Bland W (1987) Genotypic variation in crop plant root systems. Adv Agron 41:91-145

Oyanagi A, Nakamoto O, Wada M (1993) Relationship between root growth angle of seedlings and vertical distribution of roots in the field in wheat cultivars. Japan J Crop Sci 62(4):565-570

Perrier X, Jacquemoud-Collet J (2006) DARwin software. CIRAD, Montpellier, France

Phung NTP, Mai CD, Hoang GT, Truong HTM, Lavarenne J, Gonin M, Nguyen KL, Ha TT, Do VN, Gantet P, Courtois B (2016) Genome-wide association mapping for root traits in a panel of rice accessions from Vietnam. BMC Plant Biol 16(1):1-19

Preger V, Tango N, Marchand C, Lemaire SD, Carbonera D, Di Valentin M, Costa A, Pupillo P, Trost P (2009) Auxin-responsive genes AlR12 code for a new family of plasma membrane b-Type cytochromes specific to flowering plants. Plant Physiol 150(2):606-620

Pritchard JK, Stephens M, Donnelly P (2000) Inference of population structure using multilocus genotype data. Genetics 155(2):945-959

Sakurai J, Ahamed A, Murai M, Maeshima M, Uemura M (2008) Tissue and cellspecific localization of rice aquaporins and their water transport activities. Plant Cell Physiol 49(1):30-39

Sanguineti M, Li S, Maccaferri M, Corneti S, Rotondo F, Chiari T, Tuberosa R (2007) Genetic dissection of seminal root architecture in elite durum wheat germplasm. Ann Appl Biol 151(3):291-305

Storey JD, Tibshirani R (2003) Statistical significance for genomewide studies. Proc Natl Acad Sci 100(16):9440-9445

Singh V, van Oosterom EJ, Jordan DR, Hammer GL (2012) Genetic control of nodal root angle in sorghum and its implications on water extraction. Eur J Agron 42:3-10

Tian F, Bradbury PJ, Brown PJ, Hung H, Sun Q, Flint-Garcia S, Rocheford TR, McMullen MD, Holland JB, Buckler ES (2011) Genome-wide association study of leaf architecture in the maize nested association mapping population. Nat Genet 43(2):159-162

Trachsel S, Kaeppler SM, Brown KM, Lynch JP (2011) Shovelomics: high throughput phenotyping of maize (Zea mays L.) root architecture in the field. Plant Soil 341:75-87

Ueno K, Sato T (1989) Aerial root formation in rice ecotype Bulu. Japan J Trop Agric 33(3):173-175

Ueno K, Sato T (1992) Varietal difference in growth directions of rice crown roots and relation to gravitropic response and diameter of crown roots. Japan J Breed 42(4):779-786

Uga Y, Ebana K, Abe J, Morita S, Okuno K, Yano M (2009) Variation in root morphology and anatomy among accessions of cultivated rice (Oryza sativa L.) with different genetic backgrounds. Breed Sci 59(1):87-93

Uga Y, Hanzawa E, Nagai S, Sasaki K, Yano M, Sato T (2012) Identification of qSOR1, a major rice QTL involved in soil-surface rooting in paddy fields. Theor Appl Genet 124(1):75-86

Uga Y, Kitomi Y, Ishikawa S, Yano M (2015) Genetic improvement for root growth angle to enhance crop production. Breed Sci 65(2):111-119

Uga Y, Kitomi Y, Yamamoto E, Kanno N, Kawai S, Mizubayashi T (2015) A QTL for root growth angle on rice chromosome 7 is involved in the genetic pathway of DEEPER ROOTING 1. Rice 8:8

Uga Y, Okuno K, Yano M (2011) Dro1, a major QTL involved in deep rooting of rice under upland field conditions. J Exp Bot 62(8):2485-2494

Uga Y, Sugimoto K, Ogawa S, Rane J, Ishitani M, Hara N, Kitomi Y, Inukai Y, Ono K, Kanno N, Inoue H, Takehisa H, Motoyama R, Nagamura Y, Wu J, Matsumoto T, Takai T, Okuno K, Yano M (2013) Control of root system architecture by DEEPER ROOTING 1 increases rice yield under drought conditions. Nat Genet 45:1097-1102

Uga Y, Yamamoto E, Kanno N, Kawai S, Mizubayashi T, Fukuoka S (2013) A major QTL controlling deep rooting on rice chromosome 4. Sci Rep 3:3040

White PJ, George TS, Dupuy LX, Karley AJ, Valentine TA, Wiesel L, Wishart J (2013) Root traits for infertile soils. Front Plant Sci 4:193 
Yadav R, Courtois B, Huang N, McLaren G (1997) Mapping genes controlling root morphology and root distribution in a double-haploid population of rice. Theor Appl Genet 94:619-632

Zhang P, Zhong K, Shahid MQ, Tong H (2016) Association analysis in rice: from application to utilization. Front Plant Sci 7:1202

Zhao K, Tung C-W, Eizenga GC, Wright MH, Ali ML, Price AH, Norton GJ, Islam R Reynolds A, Mezey J, McClung AM, Bustamante CD, McCouch SR (2011) Genome-wide association mapping reveals a rich genetic architecture of complex traits in Oryza sativa. Nat Commun 2:467

\section{Submit your manuscript to a SpringerOpen ${ }^{\bullet}$ journal and benefit from:}

- Convenient online submission

- Rigorous peer review

- Open access: articles freely available online

- High visibility within the field

- Retaining the copyright to your article

Submit your next manuscript at $\boldsymbol{\sim}$ springeropen.com 